Cita bibliográfica: Flores Ruiz, D., Bago Sotillo, E. y Barroso González, M.O. (2018). Comportamiento del turismo nacional y crecimiento en España Comportamiento del turismo nacional y crecimiento en España. Investigaciones Turísticas (16), pp. 68-86. http://dx.doi.org/10.14198/INTURI2018.16.04

\title{
Comportamiento del turismo nacional y crecimiento en España
}

\author{
National Tourism Behavior and Growth in Spain
}

David Flores Ruiz, Universidad de Huelva, España. david.flores@dege.uhu.es Elena Bago Sotillo, Universidad de Huelva, España. elena.bago@gmail.com María de la O Barroso González, Universidad de Huelva, España. barroso@uhu.es

\section{RESUMEN}

En este artículo se estudia el comportamiento de la demanda turística de los españoles respecto al crecimiento de la economía en los primeros años de este siglo. El estudio se centra exclusivamente en la población residente en España, de forma que se analizan las relaciones de causalidad entre las variaciones del Producto Interior Bruto y las preferencias turísticas de los españoles. En primer lugar, se evalúa esta influencia sobre el flujo turístico de los residentes en España, haciendo una distinción entre el turismo emisor y el turismo interno y, en segundo lugar, las distinciones se realizan sobre el turismo rural y el resto de tipologías alojados en establecimientos hoteleros. Para ello, se utilizan datos de las series temporales trimestrales del producto interior bruto nacional, del número de viajes al extranjero, del número de viajes internos, del total de pernoctaciones en hoteles nacionales y del número de pernoctaciones en alojamientos de turismo rural. Los resultados confirman una causalidad bidireccional entre el turismo nacional y crecimiento económico, detectándose una ruptura estructural en 2008 que no afecta a las relaciones entre las variables y no producen alteraciones en las causalidades.

Palabras claves: Demanda turística; Turismo Nacional; Turismo Emisor; Turismo Interno; Crecimiento Económico.

\section{ABSTRACT}

This paper studies the behavior of the tourist demand of Spaniards in relation to the economic growth in the early years of this century. The study focuses exclusively on the resident population of Spain in order to analyze the causal relationships between the variations of the Spanish Gross Domestic Product and the tourist preferences of Spaniards. First it assesses this influence on the tourist flow of the residents in Spain distinguishing between outbound tourism and domestic tourism. A distinction between traditional tourism and rural tourism is also made. This is done using data from the quarterly national time series of the national Gross Domestic Product, the number of trips abroad, the number of internal trips, the total number 
of nights spent by domestic tourists in hotels and the number of nights spent by national tourists in rural tourism accommodation. The results confirm a bi-directional causality by detecting a structural break in 2008 that does not affect the relationships between the variables and does not produce alterations in the causalities.

Keywords: Tourist Demand; National Tourism; Outbound Tourism; Domestic Tourism; Economic Growth.

\section{INTRODUCCIÓN}

Tradicionalmente ha existido un reconocimiento insuficiente del papel estratégico de la actividad turística en el crecimiento y desarrollo económico de los territorios (Torres Bernier, 1994; Bote Gómez, 1994; Pulido, Flores, y Vargas-Machuca, M.J., 2008; entre otros). Este insuficiente reconocimiento puede deberse a enfoques teóricos inadecuados y a los prejuicios y peculiaridades de los servicios en general y la actividad turística en particular, en cuanto a actividades productivas con repercusiones en las economías de los países.

En este sentido, como reconoce Bote Gómez (1994), durante los años sesenta y setenta la estrategia mundial de desarrollo se centró esencialmente en sectores productores de bienes materiales, prestándose una insuficiente atención al sector terciario o de servicios. De hecho, el desarrollo económico de un país pasaba por la industrialización del mismo, es decir, por el desarrollo de su sector secundario, el cual debía mejorar la productividad de la economía, aportando producción y valor añadido a la misma.

En esta línea, la importancia social, y sobre todo económica, que comenzaba a tener la actividad turística, a partir de la década de los sesenta, era minimizada por importantes organismos internacionales, tales como el Banco Mundial. Así, este organismo, en un informe sobre la economía española, elaborado en 1963, consideraba que su desarrollo económico no se estaba construyendo sobre unas bases sólidas, pues se basaba, en gran medida, en el desarrollo de su sector turístico (Bote Gómez, 1998:30). En dicho informe, se consideraba a esta actividad económica como una actividad "coyuntural", al entender que el crecimiento de la demanda turística no era más que una moda, que podía decaer y que, por tanto, el desarrollo económico de un país no debía basarse en la misma.

Sin embargo, varias décadas después, tanto organismos internacionales (OECD, 2009; UNWTO, 2005; WTTC, 2005), como investigaciones que se han llevado a cabo sobre el tema (Cooper, Fletcher, Fyall, Gilbert y Wanhill, 2007; Lanza et. Al, 2003; Tribe, 2005; Sinclair, 1998, entre otros), ponen de manifiesto la importancia que tiene el turismo en el crecimiento y desarrollo económico de los países.

En este sentido, y para el caso español, las investigaciones realizadas han demostrado, que la demanda de turismo internacional hacia España es una corriente sostenida y no fácilmente desviable (Bote Gómez, 1998:31).

No en vano, ante la grave crisis económica de 2008, cuando la mayor parte de los sectores económicos, y la economía, en general, continuaban estancados, la llegada de turistas internacionales a España aumentó en 2011 un 7,6\%, recibiendo un total de 56,7 millones de turistas extranjeros. Y, ya, en 2016, el incremento fue del 10,3\%, llegando a recibir 75,6 
millones (Instituto de Estudios Turísticos, 2016), mientras que para el 2017 ese crecimiento se situó en el 8,6\%, llegando a los 82 millones (Instituto de Estudios Turísticos, 2018). Todo ello pone de manifiesto cómo la demanda de turismo internacional hacia España se recupera fácilmente de la crisis, demostrándose nuevamente ese carácter estructural, anteriormente comentado.

De hecho, para el caso español, tal y como demuestran Perles-Ribes et al. (2017), a pesar de la importante crisis económica, las relaciones entre crecimiento económico y turismo internacional se han mantenido, si bien, los resultados son más sensibles, dependiendo del tipo de variables y metodologías utilizadas.

En definitiva, como recogen Carrillo Hidalgo y Pulido Fernández (2012), la literatura económica parece haber asumido la importancia que puede adquirir el turismo en el crecimiento y desarrollo económico de los territorios. No obstante, como siguen reconociendo, se hace necesario que esos países o territorios reúnan una serie de condiciones, y que el modelo de desarrollo turístico se adapte a esas condiciones para que el turismo incida, en mayor medida, en el crecimiento y desarrollo económico de los mismos. En esta misma línea también se manifiestan Brida et al. (2008) a la hora de analizar cómo el turismo ha contribuido, de forma desigual, al crecimiento económico de España, Francia, Reino Unido y Estados Unidos.

En definitiva, por todas estas razones, los economistas, hasta épocas muy recientes, no han prestado mucha atención a los análisis teóricos y empíricos de las posibles contribuciones que puede hacer el turismo a las economías. Así como tampoco al análisis interno de la gestión eficiente y competitiva de este sector, ya que posee unas características muy específicas que lo hacen diferente, y mucho más complejo, que el resto de los sectores productivos (Pedreño dir., 1996).

Por tanto, tomando como punto de partida todas estas consideraciones, en el presente artículo se analiza: a) por un lado, el comportamiento que ha tenido la demanda turística nacional ante la evolución experimentada por el crecimiento económico del país; y, por otro, b) en qué medida ese crecimiento económico, se ha visto impulsado por el crecimiento de la demanda de turismo nacional. Para ello, como indican los estudios que se revisan en el análisis del estado de la cuestión, se parte de la hipótesis de que el turismo nacional ha influido en el crecimiento económico del país y, a su vez, el crecimiento económico ha influido en el incremento de la demanda turística nacional.

La principal originalidad de este estudio es que se centra en el análisis de la demanda turística española y su relación con el crecimiento económico del país; frente al resto de estudios que, para el caso español, han tomado en consideración la demanda turística internacional (Perles-Ribes, et al. 2017:97). En este sentido, se intenta validar si las conclusiones a las que llegan anteriores estudios en lo que respecta a las relaciones entre crecimiento económico y turismo internacional son válidas para el crecimiento nacional y la demanda turística de los españoles; pues ello podría ser un buen indicador del potencial que puede tener esta demanda a la hora de desarrollar el sector turístico español e impulsar el crecimiento económico del país. 


\section{MARCO TEÓRICO}

\subsection{Turismo y crecimiento económico}

En las últimas décadas, como consecuencia de esta importancia económica que ha comenzado a adquirir el sector turístico, han sido muchos los trabajos que se han ocupado de estudiar las relaciones que se establecen entre el turismo y el crecimiento económico, tanto a nivel internacional como a nivel de países. Estas investigaciones han intentado verificar: a) si el crecimiento turístico es causa del crecimiento económico; b) si el crecimiento económico es causa del crecimiento turístico; y/o c) si se puede llegar a identificar una interrelación entre ellos (relación bidireccional entre ambas variables).

En este sentido, la mayor parte de las investigaciones realizadas sobre ello, comparten la afirmación de la importancia económica del turismo. De hecho, son pocos los casos analizados en los que no se llega a identificar relación alguna entre turismo y crecimiento económico (Brida et al., 2013).

Así, según la revisión bibliográfica que realizan Brida et al. (2013), las relaciones de carácter unidireccional, en las que el crecimiento turístico incide en el crecimiento económico, se han puesto de manifiesto en los siguientes países: Túnez, Sudáfrica, Antigua y Barbuda, Chile, Colombia, Uruguay, México, Nicaragua, Fiji, Tonga, Islas Salomón y Papau Nueva Guinea, Taiwan, Tursquía, Italia, España, Jordania, India y Singapur.

Por su parte, siguiendo a estos mismos autores, las relaciones de carácter bidireccional se han encontrado en los siguientes países: Malasia (Lean y Tang, 2010), Corea (Cheng y Song, 2009), Taiwan (Lee y Chien, 2008; Kim et. Al, 2006), España (Cortés-Jiménez y Pulina, 2010; Mérida y Golpe, 2016; Perles-Ribes, et al. 2017), Malta (Katircioglu, 2009b), Chipre (Louca, 2006), Turquía (Demiröz y Ongan, 2005) y Grecia (Dritsakis, 2004).

En otros estudios sólo se halló una relación unidireccional desde el crecimiento económico hacia el crecimiento turístico. Estos estudios fueron los de: Tang y Jan (2009) para Estados Unidos; Oh (2005) para Corea; Narayan (2004) para Fiji; Payne y Mervar (2010) para Croacia y Katircioglu (2009b) para Chipre.

Respecto a la metodología utilizada en estos estudios, todos aplicaron la teoría de la cointegración de Johansen y luego analizaron las relaciones de causalidad mediante el test de causalidad de Granger (Granger, 1969), tanto para períodos como para países diferentes.

Sin embargo, siguiendo la misma metodología, en algunos estudios se detectan diferencias en los resultados para un mismo país. Estas diferencias se explican según la elección del período de tiempo considerado en el análisis, así como de las variables que representan al turismo y al crecimiento económico en cada uno de esos estudios.

A su vez, cuando el análisis se hace a nivel regional, los resultados para diferentes regiones de un mismo país, son también diferentes, como consecuencia de los distintos grados de especialización turística de cada una de esas regiones (Brida, et. al, 2013).

Por tanto, dado que el tipo e intensidad de las relaciones entre turismo y crecimiento económico, depende del período de tiempo considerado, de la tipología de turismo analizada y de las variables utilizadas; el estudio, que a continuación se hace, adquiere las siguientes 
características: a) analiza el caso español, a partir de un período de tiempo más actual (19992017); b) analiza diferentes tipologías de turismos (turismo interno vs turismo emisor y turismo en alojamientos hoteleros vs turismo en alojamientos rurales); y c) utiliza una metodología diferente.

Así, considerando los resultados de la mayor parte de esos estudios, como hipótesis de partida, a contrastar, se considera que el crecimiento turístico español (demanda de turismo nacional) incide en su crecimiento económico (Nowak et al. 2007; Balaguer y Cantavella-Jordá, 2002; Cortés-Jiménez y Pulina, 2010; Mérida y Golpe, 2016; Perles-Rubio, et al., 2017) y que, a su vez, el crecimiento económico incide en su crecimiento turístico (Cortés-Jiménez y Pulina, 2010; Mérida y Golpe, 2016; Perles-Ribes, et al., 2017), si bien, este grado de intensidad puede ser diferente según se trata de turismo interno, turismo emisor, en alojamientos hoteleros o en alojamientos rurales.

No obstante, previamente al análisis de los datos, se conceptualiza la demanda turística y se caracteriza el comportamiento de la demanda turística de los españoles (nacional), la cual se divide en demanda interna y demanda emisora.

\subsection{Conceptualización de la demanda turística}

Las definiciones de turismo centradas desde el lado de la demanda han evolucionado, primero intentando sintetizar la idea de turismo dentro de las definiciones "conceptuales" $y$, en segundo lugar, a través del desarrollo de definiciones "técnicas" con fines de medición y de índole legal con objeto de poder comparar datos entre países.

Desde el punto de vista conceptual, podemos concebir el turismo, según la OMT (1994), como las actividades de personas que viajan a lugares situados fuera de su entorno habitual y permanecen allí durante no más de un año seguido por motivos de ocio o negocio o por otras razones.

Por tanto, podría decirse que el consumo asociado a la demanda turística se define como el gasto realizado por, o en nombre de, el visitante, antes, durante o después del viaje, estando dicho gasto relacionado con el viaje y desarrollándose el viaje fuera del entorno habitual (INE, 1994). La demanda turística abarca tanto el desplazamiento necesario como todos los bienes y servicios requeridos por el consumidor durante dicho desplazamiento (Iranzo et al, 2003:60).

A su vez, la demanda turística se puede dividir en: a) demanda turística interna, la cual está constituida por los turistas de un país que viajan dentro del mismo país; y b) demanda turística internacional, integrada por los turistas de un país que viajan hacia otro país. Ésta, a su vez, puede diferenciarse entre demanda emisora (turistas que viajan a otro país) y demanda receptora (turistas que llegan de otros países al país de referencia).

El presente artículo analiza el comportamiento que, en los últimos años, viene experimentando el turismo de los españoles, diferenciando entre demanda interna (turistas españoles que viajan por España) y demanda emisora (turistas nacionales que viajan al extranjero). Ambas demandan conforman la demanda de turismo nacional. 
Desde un punto de vista teórico, los factores o variables que determinan y explican el comportamiento de la demanda turística, según Iranzo et al (2003), se pueden clasificar en los siguientes grupos: precios, nivel de renta disponible, hábitos de consumo, influencia de la publicidad, tiempo disponible, socio-demográficos, calidad/precio, de riesgo y macroeconómicos.

Todos estos factores, han incidido en el importante crecimiento y diversificación experimentada por la demanda turística internacional en el último medio siglo. En este sentido, puede afirmarse que este cambio esencial en el turismo tiene tanto que ver con el incremento de la demanda, como con su pluralidad, factores ambos que habrán de acentuarse en los próximos años y décadas, según la OMT en sus perspectivas hasta 2020 (OMT, 2001).

Sin embargo, podría decirse que a pesar de que la evolución de la demanda turística pueda estar determinada por todos estos factores, como recoge Esteban Talaya (2004:83), la capacidad explicativa de las variables económicas, sobre todo globales, se ha manifestado mucho más precisa, considerando que la adquisición de servicios turísticos se asemeja a un consumo total o de un conjunto de productos, además de mejor posibilidad de cuantificación de estas variables.

En definitiva, los estudios demuestran cómo buena parte del comportamiento de la demanda turística es explicado por factores o variables económicas. De tal forma que, en el presente artículo, pretendemos analizar las relaciones que se establecen entre el comportamiento de la renta en España y la demanda turística de los españoles, con objeto de demostrar las relaciones que se establecen entre las mismas y cómo el grado de intensidad de estas relaciones varía según el tipo de turismo (interno vs emisor y hotelero vs alojamiento rural). También se analiza en qué medida el crecimiento del turismo en España impulsa, y está relacionado, con el crecimiento económico del país.

\subsection{La demanda turística en nacional}

La información facilitada por la Encuesta de Turismo de Residentes, elaborada por el INE (2018), respecto a los viajes de los españoles, muestra que en el año 2017 tanto el turismo emisor como el doméstico registraron incrementos. Los residentes en España realizaron 193,7 millones de viajes, un 6,4\% superior al del año anterior, representando el turismo doméstico un $91,1 \%$ frente al emisor. Según el INE (2016), los españoles que más viajaron en 2015 fueron los residentes en la Comunidad de Madrid, seguidos de andaluces y catalanes. En cuanto al motivo, el ocio fue el principal motivo de viaje, tanto en el turista doméstico como en el emisor (47,1\% del total), seguido de las visitas a familiares o amigos (38,7\%); aunque la mayor tasa de crecimiento interanual ha sido para el turismo de negocios, tanto de españoles como de extranjeros, como consecuencia de la mejora del escenario macroeconómico en España y tras varios años marcados por recortes y planes de contingencia empresarial.

Los viajes de ocio acumularon el 55,7\% del gasto total, con un gasto medio diario de unos 57 euros. El gasto medio diario más elevado fue el de los viajes de negocios (88 euros). Los residentes optaron por alojamiento no de mercado (64,3\% del total de viajes). Estos viajes, que acumularon el $63,2 \%$ de las pernoctaciones, presentaron el gasto medio diario más bajo 
(28 euros). El gasto medio diario más elevado (116 euros) fue el de los viajes en los que se utilizó un alojamiento hotelero.

En cuanto al turismo doméstico, pese a la recesión que ha experimentado en los últimos años debido a la crisis económica, sigue imponiéndose de manera notable sobre el turismo emisor. De forma que el $91,1 \%$ de los viajes tuvo como destino principal España. Según el INE (2016), los viajes dentro de España supusieron el 82,2\% de las pernoctaciones, con un gasto total de más de 26 millones de euros, lo que supone un incremento superior al 5\% respecto al año 2014 , correspondiente al $68 \%$ del gasto total de los españoles en turismo. El gasto medio diario fue de 42 euros y recayó principalmente en sectores asociados indirectamente con el turismo, como son bares, restaurantes y transporte. El alojamiento fue el tercer subsector donde fue a parar el gasto del turista doméstico. Andalucía fue el destino más visitado con un $17,3 \%$ de los viajes, seguida de Cataluña y Comunidad Valenciana. Los gastos medios diarios más altos fueron los de Canarias y Comunidad de Madrid.

Respecto al turismo emisor, según el Instituto de Estudios Turísticos (2016), el 8,6\% de los turistas residentes en España viajaron al extranjero en 2015. Los viajes al extranjero se incrementaron en un $2 \%$ suponiendo el $17,8 \%$ de las pernoctaciones y el $31,4 \%$ del gasto total en turismo y un gasto medio diario de unos 90 euros que recayó principalmente y por este orden en transporte, bares y restaurantes y alojamiento.

Por tanto, cuando hablamos de turismo interno y turismo emisor estamos haciendo referencia a dos tipologías de turismo diferentes, con un gasto medio diario, un gasto total y una estancia media, que difieren sustancialmente, a favor del turismo emisor, el cual puede ser considerado como de categoría superior. Ello podrá incidir en un comportamiento diferente respecto al crecimiento económico y, por tanto, de la renta nacional, lo cual se pone de manifiesto en el análisis empírico.

Por su parte, si distinguimos en función del tipo de alojamiento, entre alojamientos hoteleros y alojamientos de turismo rural, podría decirse que, en los últimos años, el turismo rural está cobrando gran importancia ganando adeptos entre los residentes españoles. No en vano, el número de turistas residentes alojados en establecimientos rurales en España subió, en 2017 , un $8,8 \%$, frente al $1,4 \%$ de los que optaron por alojamientos hoteleros (INE, 2018b).

\section{METODOLOGÍA DEL ESTUDIO}

El principal objetivo de este trabajo es el de analizar los efectos de causalidad que se establecen entre el comportamiento del turismo nacional y el crecimiento económico de España, con objeto de poner de manifiesto la importancia que tiene este tipo de demanda frente a la demanda internacional. Para ello, se analizarán las relaciones de causalidad entre las variaciones del Producto Interior Bruto español y de la demanda turística española, en la cual identificaremos la demanda de turismo interno y de turismo emisor, así como las de turismo hotelero y en alojamientos rurales ${ }^{1}$.

1. Se están dejando fuera las ocupaciones en camping, apartamiento y viviendas turísticas, si bien el objetivo de este análisis es contrastar si existen diferencias entre diferentes tipos de turismo, para lo cual se ha considerado oportuno elegir el turismo rural y el turismo hotelero que no se encuentra en el medio rural. 
Para medir la demanda turística, las variables cuantitativas han sido, tradicionalmente: a) en unidades físicas, el número de viajes y el número de pernoctaciones, y b) en unidades monetarias, el gasto turístico, es decir, todo gasto de consumo efectuado por un visitante durante y para su desplazamiento y permanencia turística en el lugar de destino. En nuestro estudio no utilizaremos variables monetarias con el fin de evitar problemas de multicolinealidad, por lo que nos detendremos en analizar el número de viajes y las pernoctaciones del turista español.

El número de viajes de los residentes españoles la dividiremos entre turismo interno y turismo emisor. De esta forma, además de estudiar la relación entre el crecimiento económico y el número de viajes de los españoles podemos observar si existen diferencias significativas en la influencia que ejerce la situación económica del país respecto a los distintos tipos de viajes realizados: turismo interno y turismo emisor.

A su vez, el turismo interno lo dividiremos en turismo en alojamientos rurales y en alojamientos hoteleros, con objeto de detectar si ambos tipos experimentan comportamientos diferentes según la evolución económica del país.

Por su parte, para cuantificar el crecimiento económico se utilizará como indicador la evolución del producto interior bruto español a precios corrientes.

Los datos utilizados proceden del Instituto Nacional de Estadística, para el caso del PIB. El número de pernoctaciones en establecimientos hoteleros proceden de la Encuesta de Ocupación Hotelera (EOH) y el número de pernoctaciones en alojamientos de turismo rural de la Encuesta de Ocupación en Alojamientos Rurales, ambas de la base de datos del Instituto Nacional de Estadística. Por otra parte, los datos sobre los viajes de los residentes en España proceden de la Encuesta sobre Turismo de Residentes, publicada por el INE a partir de febrero de 2015 y anteriormente por FAMILITUR. (Para su adaptación a los cambios de metodología el INE utiliza un procedimiento de enlace).

Todos los datos se agrupan en series temporales con periodicidad trimestral siendo el primer dato el correspondiente al primer trimestre de 1999 (1Q1999) y el último el correspondiente al tercer trimestre de 2017 (3Q2017).

Para detectar el tipo de causalidad que se establece entre la demanda turística nacional y el crecimiento económico del país se calcula el coeficiente de correlación cruzada entre cada una de las variables (PIB, para el crecimiento, y número de viajes y pernoctaciones para la demanda turística). No obstante, previamente se realiza el test de contraste de ruptura estructural, a través del test de estabilidad de Chow (1960), para ver si las series siguen un patrón de comportamiento uniforme en el largo plazo.

Se sigue el análisis de causalidad de Pierce y Haugh, que consiste en identificar y estimar el modelo ARIMA que mejor se ajuste a cada serie, obteniendo una serie preblanqueada, formada por los residuos ruido blanco en la que se ha eliminado cualquier tipo de autocorrelación. Para finalizar, se estima la correlación cruzada entre las series preblanqueadas y se determinan las relaciones de causalidad entre cada par de variables.

La técnica de correlación cruzada fue introducida por Box y Jenkins (1970) e inicialmente utilizada como una técnica estadística para especificar un modelo de función de transferencia. 
Pierce y Haugh (1977) la utilizan como medida de causalidad en el sentido de Granger para lo cual realizan diversos ajustes.

Esta técnica ha sido poco utilizada, predominado técnicas más sofisticadas como los modelos de vectores autoregresivos (VAR), los modelos estructurales de vectores autoregresivos (SVAR) y los modelos de vectores de corrección de errores (VEC). Sin embargo es una técnica menos compleja en su desarrollo, más intuitiva para su análisis y permite inferir relaciones de causalidad estadística del tipo instantánea, unidireccional y bidireccional a diferencia de la causalidad propuesta por Granger, que no permite esta inferencia.

La técnica de correlaciones cruzadas puede subestimar la relación causal entre variables puesto que la correlación cruzada no se obtiene de los valores de las propias variables sino de la serie preblanqueada constituida por los residuos ruido blanco. Esto hace que puedan darse autocorrelaciones significativas en determinados retardos. Mark (1979, citado por Arnau 1995, p. 159) comenta que "la correlación cruzada constituye un poderoso instrumento estadístico para la inferencia de la causalidad".

\section{RESULTADOS}

En primer lugar, para estandarizar medidas y evitar problemas de heterocedasticidad, las series con los datos brutos se han transformado tomando logaritmos neperianos y se han ajustado de manera que no se vean afectadas por la estacionalidad, separando la tendencia del ciclo.

La extracción de una señal cíclica consiste en buscar un filtro adecuado, que transforme la serie original en una señal de crecimiento. Para ello existen una gran variedad de procedimientos, como puede verse en Canova (1998). Esta diversidad de métodos se justifica diciendo que el ciclo económico puede no ser robusto al método de extracción utilizado. Sin embargo, algunos autores como Canova (1998) y Burnside (1998) encuentran que las propiedades cíclicas de las variables son robustas a la elección del método de filtración. Únicamente en el largo plazo puede haber modificaciones en las fluctuaciones que llevan a cambios en las propiedades básicas del ciclo: volatilidad, co-movimiento y persistencia, según Agenor et al. (2000) y Male (2010).

En este sentido, tanto las series originales (en logaritmos), para observar las tendencias a largo plazo, como sus ciclos, con objeto de observar comportamientos en el corto plazo, se representan en la figura 1. (ver Apéndice).

\subsection{Test de contraste de ruptura estructural}

Un análisis cualitativo de los ciclos de las series nos indica que el ciclo no sigue un patrón uniforme desde el punto de vista de largo plazo. En todos los ciclos de las series estudiadas se aprecian dos etapas, marcadas por la crisis económica, en la evolución de las fluctuaciones que tienen como consecuencia que la media y la varianza difieran entre una etapa y otra, dando lugar a un proceso estocástico no estacionario. Para corroborar la existencia de este cambio estructural se impone, en primer lugar, un punto de ruptura en la mitad de la muestra 
estudiada (2008), que viene marcado por el año de comienzo de la crisis, y se testean los coeficientes de cada fracción de tiempo a través del test de estabilidad de Chow (1960).

Los resultados del test, muestran un cambio estructural en las relaciones existes entre la evolución económica y las variables turísticas. De forma que el número de viajes al extranjero de los residentes en España experimenta un cambio durante el año 2011, produciéndose un cambio similar para el número de viajes internos durante el año 2008 aunque menos acusado que el anterior. Para el caso de las pernoctaciones, éstas sufrieron un cambio estructural en 2008 en alojamientos hoteleros y en 2011 en alojamientos de turismo rural, siendo más acusado en el primer caso.

\subsection{Volatilidad de las series}

Para un análisis más completo de los ciclos se calculan las volatilidades absolutas del componente cíclico de cada serie mediante la desviación estándar de la variable de interés. La volatilidad de una variable nos indica cuánto la misma tiende a alejarse de su tendencia de largo plazo. Cuanto mayor sea este coeficiente, la variable será más volátil. La medida de la volatilidad relativa respecto al ciclo económico se aproximada mediante el cociente entre la volatilidad estándar del ciclo de cada serie y la desviación estándar del ciclo del PIB. Si la volatilidad relativa es superior a la unidad significa que la variable presenta mayor amplitud cíclica que el producto (Male, 2010:8).

Tabla 1. Media, desviación estándar y volatilidades para los ciclos de viajes nacionales respecto al ciclo de crecimiento económico

\begin{tabular}{|c|c|c|c|c|c|c|}
\hline & N & Mínimo & Máximo & Media & $\begin{array}{c}\text { Desviación } \\
\text { estándar }\end{array}$ & Varianza \\
\hline Ciclo_PIB & 72 &,- 00158 &, 00121 &, 0000054 &, 00064005 &, 000 \\
\hline Ciclo_turismo_emisor & 72 &,- 04706 &, 03213 &, 0001169 &, 01376667 &, 000 \\
\hline Ciclo_turismo_interno & 72 &,- 02395 &, 02069 &,- 0000216 &, 00881347 &, 000 \\
\hline Ciclo_turismo_nacional & 72 &,- 02519 &, 02069 &,- 0000262 &, 00894759 &, 000 \\
\hline N válido (por lista) & 72 & & & & & \\
\hline
\end{tabular}

Fuente: elaboración propia a partir del Instituto de Estudios Turísticos (2016), INE (2018a).

Tabla 2. Media, desviación estándar y volatilidades para los ciclos de pernoctaciones respecto al ciclo de crecimiento económico

\begin{tabular}{|c|c|c|c|c|c|c|}
\hline & N & Mínimo & Máximo & Media & $\begin{array}{c}\text { Desviación } \\
\text { estándar }\end{array}$ & Varianza \\
\hline Ciclo_PIB & 64 &,- 00158 &, 00121 &, 0000054 &, 000640065 &, 000 \\
\hline Ciclo_ocupación_hotelera & 64 &,- 00746 &, 00828 &, 0000165 &, 00338189 &, 000 \\
\hline Ciclo_ocupacion_rural & 64 &,- 02465 &, 02166 &, 0001706 &, 01069771 &, 000 \\
\hline $\begin{array}{c}\text { Ciclo_suma_ocupacion_hotelera_ } \\
\text { rural }\end{array}$ & 64 &,- 00771 &, 00865 &, 0000145 &, 00358043 &, 000 \\
\hline N válido (por lista) & 64 & & & & & \\
\hline
\end{tabular}

Fuente: elaboración propia a partir del Instituto de Estudios Turísticos (2016), INE (2018b). 
Si calculamos las volatilidades relativas para el periodo completo de las series obtenemos que todas son superiores a la unidad. El turismo emisor presenta una mayor volatilidad relativa, respecto al PIB, que el turismo interno. De la misma forma, las pernoctaciones en alojamientos rurales también presentan una mayor volatilidad que las pernoctaciones en alojamientos hoteleros. Observando las medias y las desviaciones típicas de cada una de las series, podemos subdividir el periodo completo en cuatro sub-periodos, con medias y varianzas que difieren entre sub-periodos.

Tabla 3. Media, desviación estándar y volatilidades para los ciclos de viajes nacionales respecto al ciclo de crecimiento económico, considerando sub_periodos

\begin{tabular}{|c|c|c|c|c|c|c|c|c|}
\hline \multirow{2}{*}{ Periodo } & \multicolumn{2}{|c|}{ Ciclo_PIB } & \multicolumn{2}{c|}{ Ciclo_turismo_emisor } & \multicolumn{2}{c|}{ Ciclo_turismo_interno } & \multicolumn{2}{c|}{ Ciclo_turismo_nacional } \\
\cline { 2 - 10 } & Media & $\begin{array}{c}\text { Desviación } \\
\text { estándar }\end{array}$ & Media & $\begin{array}{c}\text { Desviación } \\
\text { estándar }\end{array}$ & Media & $\begin{array}{c}\text { Desviación } \\
\text { estándar }\end{array}$ & Media & $\begin{array}{c}\text { Desviación } \\
\text { estándar }\end{array}$ \\
\hline $1999-2005$ &, 0000399 &, 00050293 &, 0005992 &, 02116301 &, 0000382 &, 01226967 &, 0000437 &, 01259881 \\
\hline $2006-2007$ &, 0002124 &, 00080823 &, 0006885 &, 00771841 &, 0001292 &, 00440623 &, 0001679 &, 00446273 \\
\hline 2008 &, 0006572 &, 00038608 &,- 0038476 &, 00983910 &,- 0004611 &,- 00299143 &,- 0006765 &, 00346439 \\
\hline $2009-2017$ &,- 0000902 &, 00067514 &, 0001916 &, 00678476 &,- 0000916 &, 00692179 &,- 0000728 &, 00686025 \\
\hline
\end{tabular}

Fuente: elaboración propia a partir del Instituto de Estudios Turísticos (2016), INE (2018a).

Tabla 4. Media, desviación estándar y volatilidades para los ciclos de pernoctaciones respecto al ciclo de crecimiento económico, considerando sub_periodos

\begin{tabular}{|c|c|c|c|c|c|c|c|c|}
\hline \multirow{2}{*}{ Periodo } & \multicolumn{2}{|c|}{ Ciclo_PIB } & \multicolumn{2}{|c|}{ Ciclo_ocupacion_hotel } & \multicolumn{2}{|c|}{ Ciclo_ocupación_rural } & \multicolumn{2}{|c|}{$\begin{array}{c}\text { Ciclo_suma_ocupacion_hotelera_ } \\
\text { rural }\end{array}$} \\
\hline & Media & $\begin{array}{c}\text { Desviación } \\
\text { estándar }\end{array}$ & Media & $\begin{array}{c}\text { Desviación } \\
\text { estándar }\end{array}$ & Media & $\begin{array}{c}\text { Desviación } \\
\text { estándar }\end{array}$ & Media & Desviación estándar \\
\hline 2001-2002 &,- 0001050 & ,00033070 & ,0000139 & ,00489942 & , 0007877 & ,01580216 &,- 0000483 & ,00507309 \\
\hline $2003-2007$ & ,0000619 &, 00066260 &,- 0000927 & ,00233494 & ,0000436 & ,01059034 &,- 0000750 & ,00258310 \\
\hline 2008 & ,0006572 & ,00038608 & ,0007618 & ,00485569 & ,0005066 & ,01436804 & ,0007431 & ,00526347 \\
\hline $2009-2017$ &,- 0000852 & ,00068241 &,- 0000064 & ,00358331 & ,0001481 & ,00985854 &,- 0000075 & 00377067 \\
\hline
\end{tabular}

Fuente: elaboración propia a partir del Instituto de Estudios Turísticos (2016), INE (2018b).

\subsection{Co-movimiento de las series}

Para medir el co-movimiento de las series calculamos los coeficientes de correlación cruzada adelantados y atrasados hasta cuatro períodos trimestrales. La función de correlación cruzada, $\rho_{x y}(k)$, de dos procesos estocásticos conjuntamente estacionarios, $x_{t}, y_{t}$ viene dada por:

$$
\rho_{x y}(k)=\gamma_{x y}(k) / \sigma_{x} \sigma_{y}(K=0,+1,+2 \ldots-1,-2 \ldots)
$$

Esta función es simplemente la estandarización de la función de covarianzas y, tiene propiedades las siguientes propiedades: 
1. $\left|\rho_{x y}(k)\right| \leq 1$;

2. En general, $\rho_{x y}(k) \neq \rho_{y x}(-k)$, y la función no es simétrica alrededor del origen.

El término $\gamma_{x y}(0)$ indica la relación contemporánea o instantánea entre las variables $x, y$. Cuando este valor es cero no existe relación entre ambas variables. Los coeficientes de correlación estarán siempre entre 1, -1. El signo de la correlación indica que las variables se mueven en la misma dirección (positivo) o en direcciones opuestas (negativo). Si las variables son estadísticamente independientes el signo de la correlación tiende a cero.

Atendiendo a las definiciones de Fiorito y Kochintezas (1993), dos series son procíclicas, es decir, su movimiento se produce en el mismo sentido, si su coeficiente de correlación contemporáneo es mayor o igual a 0,2, anticíclica si es menor o igual a -0,2 y acíclica en caso contrario. La correlación será débil cuando el valor absoluto de dicho coeficiente de correlación se sitúa entre 0,2 y 0,5 y fuerte cuando es mayor a 0,5.

Tabla 5. Coeficientes de correlación cruzados del ciclo económico respecto a los ciclos de las series de turismo_nacional

\begin{tabular}{|c|c|c|c|c|c|c|}
\hline \multicolumn{7}{|c|}{ Correlaciones cruzadas } \\
\hline \multicolumn{2}{|c|}{ ciclo_turismo_emisor con ciclo_PIB } & \multicolumn{2}{c|}{$\begin{array}{c}\text { ciclo_turismo_interno con } \\
\text { ciclo PIB }\end{array}$} & \multicolumn{2}{c|}{ ciclo_turismo_nacional con ciclo_ $^{\text {PIB }}$} \\
\hline Retardo & $\begin{array}{c}\text { Correlación } \\
\text { cruzada }\end{array}$ & $\begin{array}{c}\text { Error } \\
\text { estándar }\end{array}$ & $\begin{array}{c}\text { Correlación } \\
\text { cruzada }\end{array}$ & $\begin{array}{c}\text { Error } \\
\text { estándar }\end{array}$ & $\begin{array}{c}\text { Correlación } \\
\text { cruzada }\end{array}$ & Error estándar $^{\text {a }}$ \\
\hline-12 &, 387 &, 129 &, 398 &, 129 &, 412 &, 129 \\
\hline-11 &,- 063 &, 128 &,- 198 &, 128 &,- 195 &, 128 \\
\hline-10 &,- 440 &, 127 &,- 443 &, 127 &,- 455 &, 127 \\
\hline-9 &, 264 &, 126 &, 317 &, 126 &, 320 &, 126 \\
\hline-8 &, 304 &, 125 &, 378 &, 125 &, 386 &, 125 \\
\hline-7 &,- 241 &, 124 &,- 286 &, 124 &,- 291 &, 124 \\
\hline-6 &,- 270 &, 123 &,- 340 &, 123 &,- 347 &, 123 \\
\hline-5 &, 213 &, 122 &, 248 &, 122 &, 253 &, 122 \\
\hline-4 &, 216 &, 121 &, 335 &, 121 &, 336 &, 121 \\
\hline-3 &,- 198 &, 120 &,- 337 &, 120 &,- 333 &, 120 \\
\hline-2 &,- 120 &, 120 &,- 216 &, 120 &,- 218 &, 120 \\
\hline-1 &, 177 &, 119 &, 239 &, 119 &, 241 &, 119 \\
\hline 0 &, 051 &, 118 &, 282 &, 118 &, 270 &, 118 \\
\hline 1 &,- 074 &, 119 &,- 280 &, 119 &,- 267 &, 119 \\
\hline 2 &,- 136 &, 120 &,- 243 &, 120 &,- 241 &, 120 \\
\hline 3 &, 172 &, 120 &, 306 &, 120 &, 301 &, 120 \\
\hline 4 &, 040 &, 121 &,- 229 &, 121 &, 218 &, 121 \\
\hline 5 &,- 108 &, 122 &,- 336 &, 122 &,- 320 &, 122 \\
\hline 6 &,- 087 &, 123 &,- 121 &, 123 &,- 126 &, 123 \\
\hline 7 &, 145 &, 124 & -215 &, 124 &, 214 &, 124 \\
\hline 8 &, 020 &, 125 &, 188 &, 125 &, 177 &, 125 \\
\hline 9 &,- 102 &, 126 &,- 273 &, 126 &,- 260 &, 126 \\
\hline
\end{tabular}




\begin{tabular}{|l|l|l|l|l|l|l|}
\hline 10 &,- 048 &, 127 &,- 108 &, 127 &,- 108 &, 127 \\
\hline 11 &, 099 &, 128 &, 199 &, 128 &, 194 &, 128 \\
\hline 12 &, 026 &, 129 &, 118 &, 129 &, 111 &, 129 \\
\hline
\end{tabular}

Fuente: elaboración propia a partir del Instituto de Estudios Turísticos (2016), INE (2018a).

a Se basa en el supuesto que las series no están correlacionadas de forma cruzada y que una de las series es de ruido blanco.

Tabla 6. Coeficientes de correlación cruzados del ciclo económico respecto a los ciclos de las series de pernoctaciones

\begin{tabular}{|c|c|c|c|c|c|c|}
\hline \multicolumn{7}{|c|}{ Correlaciones cruzadas } \\
\hline \multicolumn{3}{|c|}{$\begin{array}{c}\text { ciclo_ocupacion_hotelera con } \\
\text { ciclo_PIB }\end{array}$} & \multicolumn{2}{|c|}{$\underset{\text { PIB }}{\text { ciclo_ocupacion_rural ciclo_ }}$} & \multicolumn{2}{|c|}{$\begin{array}{c}\text { ciclo_suma_ocupacion_hotelera_ } \\
\text { rural ciclo PIB }\end{array}$} \\
\hline Retardo & $\begin{array}{l}\text { Correlación } \\
\text { cruzada }\end{array}$ & $\begin{array}{l}\text { Error } \\
\text { estándara }\end{array}$ & $\begin{array}{l}\text { Correlación } \\
\text { cruzada }\end{array}$ & Error estándara & $\begin{array}{l}\text { Correlación } \\
\text { cruzada }\end{array}$ & Error estándara \\
\hline-12 & ,036 & 139 &,- 110 & 139 & ,030 & 139 \\
\hline-11 &,- 086 & 137 & 210 & 137 &,- 044 & 137 \\
\hline-10 &,- 134 & 136 &,- 197 & 136 &,- 160 & 136 \\
\hline-9 & ,089 & 135 & ,028 & 135 & ,080 & 135 \\
\hline-8 & 175 & 134 & ,064 & ,134 & 176 & ,134 \\
\hline-7 &,- 161 & 132 & ,026 & 132 &,- 132 & 132 \\
\hline-6 &,- 107 & 131 &,- 097 & ,131 &,- 124 & ,131 \\
\hline-5 & 132 & 130 & ,035 & 130 & 116 & 130 \\
\hline-4 & 129 & 129 &,- 019 & 129 & 125 & 129 \\
\hline-3 &,- 272 & 128 & ,005 & 128 &,- 234 & 128 \\
\hline-2 & ,085 & 127 & ,092 & 127 & ,077 & 127 \\
\hline-1 & 156 & 126 &,- 017 & 126, & 127, & 126 \\
\hline 0 & 116 & 125 &,- 067 & 125 & 108 & 125 \\
\hline 1 &,- 176 & 126 & ,084 & 126 &,- 137 & 126, \\
\hline 2 & -,125 & 127 &,- 078 & 127, & -135 & 127, \\
\hline 3 & 098 & 128 &,- 047 & 128 & ,076 & 128 \\
\hline 4 & 234 & 129, & 156 & 129, & 240 & 129, \\
\hline 5 &,- 285 & 130, &,- 067 & 130 &,- 260 & 130 \\
\hline 6 &,- 072 & 131 &,- 045 & 131 &,- 075 & 131 \\
\hline 7 & 163 & 132 &,- 004 & 132 & 139 & 132 \\
\hline 8 & 090 & 134 & 142 & 134 & 104 & 134 \\
\hline 9 & -160 & 135 &,- 118 & 135 & -155 & 135 \\
\hline 10 &,- 085 & 136 &,- 048 & 136 &,- 084 & 136 \\
\hline 11 & 163 & 137, & 070, & 137, & 152 & 137, \\
\hline 12 &,- 013 & 139, & ,045 & 139, &,- 004 & 139, \\
\hline
\end{tabular}

Fuente: elaboración propia a partir del Instituto de Estudios Turísticos (2016), INE (2018b).

A Se basa en el supuesto que las series no están correlacionadas de forma cruzada y que una de las series es de ruido blanco. 
De acuerdo con los resultados, y en un primer análisis, podemos decir que el turismo interno, al igual que el turismo nacional son variables procíclicas respecto a los ciclos económicos existiendo una correlación débil entre ambas y comportándose el turismo emisor como una variable acíclica respecto al Producto Interior Bruto. En cuanto a las variables que cuantifican la ocupación en alojamientos ninguna de ellas presenta una correlación significativa con respecto a los ciclos económicos, permaneciendo de manera acíclica. En cuanto al desfase de los ciclos, puede observarse como el ciclo económico está adelantado en más de dos años con respecto a los ciclos turísticos que miden número de viajes. Sin embargo, este adelanto es mucho menor si nos referimos a variables que cuantifican las pernoctaciones en alojamientos hoteleros.

\subsection{Causalidad de Pierce y Haugh}

La función de correlación cruzada para $k>0$ mide la relación desde $x$ hacia $y$, mientras que para $k<0$ la relación desde $y$ hacia $x$. De esta forma, si Si $x_{t}$ es ruido blanco:

a. Para $k>0$ los coeficientes $\gamma_{x y}(k)$ representan cómo los valores de $X_{t}$ influyen en los valores futuros $Y_{t+k}$, es decir, que la parte positiva de esta función representa la relación causal de $X_{t}$ hacia $Y_{t+k}$.

b. Para $k<0$ los coeficientes $\gamma_{x y}(k)$ representan cómo los valores de Yt influyen en los valores futuros $X_{t+k}$, es decir, que la parte negativa de esta función representa la relación causal de $\mathrm{Y}_{\mathrm{t}}$ hacia $\mathrm{X}_{\mathrm{t}+\mathrm{k}}$.

Si la variable $x_{t}$ no es ruido blanco, este contraste puede aplicarse utilizando los residuos del modelo univariante de $x_{t}$, con la técnica de preblanqueado de series (Peña, 2010, p. 507). El análisis de causalidad propuesto por Pierce y Haugh (1977) utiliza la técnica de preblanqueo para eliminar cualquier patrón de autocorrelación existente en los datos.

Observando los resultados del contraste, podemos deducir, cómo el ciclo económico influye de manera considerable en el turismo nacional, siendo mayor esta influencia en el turismo interno que en el emisor. De manera bidireccional, también existe causalidad entre el turismo y el aumento del PIB, aunque ésta sólo se aprecia en el caso del turismo interno. De hecho, el turismo emisor, desde el punto de vista teórico, no aporta al PIB nacional. En cuanto a las pernoctaciones, el ciclo económico también ejerce influencia en el número de pernoctaciones en alojamientos hoteleros, pero ésta es bastante débil para el caso del alojamiento rural. Aunque también en el caso de las pernoctaciones se comprueba relación bidireccional, ésta es inferior que en el caso de los viajes internos.

En definitiva, el número de viajes del turismo interno está fuertemente influido por el crecimiento económico, siendo esta influencia menor para el caso de los viajes del turismo emisor. Al mismo tiempo, se demuestra como el desarrollo del turismo interno influye, a su vez, en el crecimiento económico de España. De tal forma que nos encontramos ante dos relaciones de causalidad que se retroalimentan. 


\section{CONCLUSIONES}

En este artículo se ha pretendido establecer unas relaciones de causalidad entre la evolución económica de España durante el actual siglo y la influencia que ha ejercido en la demanda del turismo de residentes, con objeto de poner de manifiesto el papel que, frente a la demanda turística internacional, puede desempeñar ésta. Para analizar esta influencia de manera bidireccional, se han utilizado datos de series temporales que cuantifican a la demanda a través del número de viajes y de las pernoctaciones, diferenciando entre turismo emisor e interno, así como entre tipos de alojamiento, hotelero o rural.

El primer proceso ha sido desestacionalizar las series, filtrándolas y suavizándolas, de forma que se obtenga la componente cíclica de cada una de ellas. El análisis gráfico de las componentes cíclicas indica dos periodos diferentes en cuanto a varianzas y medias de los ciclos, por lo que se ha contrastado la hipótesis de ruptura estacional mediante el test de Chow (1960). Los resultados de este test muestran, tal y como ya apuntaban Perles-Ribes (2017), también para el caso español, a una ruptura estructural para el número de viajes internos y de pernoctaciones en alojamientos hoteleros para el año 2008. Dicha ruptura, aparece también, aunque menos acusada, en el año 2011 para el caso de viajes al extranjero y pernoctaciones en alojamientos de turismo rural.

El estudio de las volatilidades relativas, muestran cómo, aunque todas las variables son volátiles respecto al Producto Interior Bruto, las variables correspondientes al turismo interno y a las pernoctaciones en alojamientos hoteleros son menos volátiles, respecto al ciclo económico, que las variables que cuantifican el turismo emisor y la ocupación en alojamientos rurales.

El co-movimiento de las series y de la causalidad, indican una causalidad fuerte del ciclo económico que influye en el turismo nacional en los dos años posteriores, especialmente en el turismo interno, que se ve más afectado por el PIB que el turismo emisor. El ciclo económico influye también de manera considerable en la ocupación de alojamientos hoteleros y débilmente en las de alojamientos rurales. Las relaciones de causalidad aparecen de manera bidireccional en el caso de turismo interno y de pernoctaciones en alojamientos hoteleros, lo cual sitúa al turismo interno en los últimos años como fuente generadora de riqueza, al igual que, según otros estudios, ocurre con la demanda de turismo internacional.

No en vano, dado que, como comentábamos en el marco teórico, el turismo interno, respecto al turismo emisor, se puede caracterizar como un tipo de turismo de categoría inferior, su evolución es más sensible a los cambios en el nivel de renta y, por tanto, a la evolución del crecimiento económico.

Por tanto, se ratifican las hipótesis de partida, en la medida en que, para el caso español, al igual que se ha puesto de manifiesto en estudios anteriores, centrados en el análisis de las relaciones entre PIB nacional y demanda turística internernacional, se concluye que existe una relación fuerte de causalidad, de carácter bidireccional, entre el turismo nacional, fundamentalmente, el turismo interno, y el crecimiento económico del país.

Todo ello pone de manifiesto la importancia que este tipo de demanda viene adquiriendo en el Siglo XXI a la hora de contribuir al crecimiento económico del país. Una demanda que, salvo en las últimas décadas, históricamente, no se le ha prestado atención alguna, tanto en el ámbito de la investigación como en el de la política económica. 
No obstante, como limitaciones de la investigación cabrían citar: a) que, aunque el objetivo fundamental es el de establecer una relación de causalidad entre el crecimiento económico en España y el turismo nacional, para demostrar el potencial que tiene esta demanda, se puede completar introduciendo la demanda de turismo internacional, con objeto de considerar el efecto global y por separado de ambas; b) así como el hecho de la falta de datos, ya que la encuesta de ocupación en alojamientos de turismo rural no comienza a publicarse por el INE hasta 2001 y la Encuesta sobre turismo de Residentes (ETR) hasta 1999.

\section{REFERENCIAS BIBLIOGRÁFICAS}

Agenor, P.R.; McDermott, C.J. y Prasad, E.S. (2000). Macroeconomic Fluctuations in Developing Countries: Some Stylized Facts", World Bank Econ Rev, 14 (2): 251-285.

Ariza Garzon, M.J. y Cadena Lozano, J.B. (2014). Identificación de relaciones entre variables de política económica en Colombia a través de funciones de correlación cruzada. Cuadernos de Administración [online], 30 (51), 36-48.

Balaguer, J. Y Cantavella-Jordá, M. (2002). Tourism as a long-run economic grown facto: The Spanis case, Applied Economic, 34, 877-884.

Brida, J.G. et al. (2008). La contribución del turismo al crecimiento económico, Cuadernos de Turismo, 22, 35-46.

Brida, J.G., Pereyra, J.S., Pulina, M., \& Such-Devesa, M.J. (2013). Causalidad entre turismo y crecimiento económico de largo plazo: una revisión crítica de la literatura econométrica, Innovar, 23(47), 53-64.

Bote Gómez, V. (1998). El turismo en España: cambio de rumbo y oportunidades científicas, Revista Valencia D'Estudis Autonomics, 25, 29-45.

Bote Gómez, V. (1994). Turismo y desarrollo económico en España: del insuficiente reconocimiento estratégico a la revalorización de su función estratégica, Papers de Turisme, 14-15, 117-129.

Box, G.E.P. y Jenkins, D.A. (1970). Distribution of the residual autocorrelations in autoregressive-integrated moving average times series models, J. Amer. Statist, 65, 1509-1526.

Bumside, C. (1998). Detrending and business ocycle facts: A comment, Journal of Monetary Economic, 41(3), 513-532.

Canova, F. (1998): Detrending and business cycle facts, Journal of Monetary Economics, 41(3), 475-512.

Carrillo Hidalgo, I. y Pulido Fernández, J.I. (2012). Análisis del papel de los organismos financieros internacionales en la financiación del turismo, Revista de Economía Mundial, 31, 49-76.

Chow, G. C. (1960): Tests of Equality Between Sets of Coefficients in Two Linear Regressions, Econométrica, 28(3), 591-605.

Cooper, C.; Fletcher, J.; Fyall, A.; Gilbert, D. y Wanhill, S. (2007): El turismo. Teoría y práctica, Madrid, Editorial Síntesis.

Cortés-Jiménez, I. y Pulina, M. (2010). Inbound tourism and long run economic growth, Current Issues in Tourism, 13 (1), 61-74.

Esteban Talaya, A. (2004). Modelos de la demanda turística en España: segmentación por país de procedencia, Mediterráneo Económico, 5, 81-101. 
Fiorito, R. y Kollintzas, T. (1994). Stylized Facts of Business Cycles in the G7 from a Real Business Cycles Perspective, European Economic Review, 38 (2), 235-269,

Granger, C. W. (1969). Investigating Cuasal Relations by Econometric Models and Cross-spectral Methods, Econometrica, 37 (3), 424-438.

INE (2016). Encuesta de turismo de residentes (ETR/Familitur), Instituto Nacional de Estadística, Madrid.

INE (2018a). Movimiento turístico en frontera, Instituto Nacional de Estadística, en http:// www.ine.es/jaxiT3/Datos.htm?t=23982. (consultada el 19 de junio 2018).

INE (2018b). Encuesta de Ocupación Hotelera y Encuesta de Ocupación, en Alojamientos Rurales, Instituto Nacional de Estadística, http://www.ine.es/dyngs/INEbase/es/categoria.htm?c=Estadistica P\&cid=1254735570703, consultada el 21 de junio de 2018).

Instituto de Estudios Turísticos (2016). Encuesta FRONTUR, Instituto de Estudios Turístico, en http://www.iet.tourspain.es

Iranzo, J.E. et ál. (2003). La demanda de turismo, en Irazo, J.E.: La estructura económica de los mercados turísticos, Instituto de Estudios Económicos, Madrid, 59-93.

Lanza, A.; Temple, P. y Urga, G. (2003). The implications of Tourism Specialisation in the Long Run: An Econometric Analysis for 13 OECD Economies, Tourism Management, 24, 315-321.

Male, R. (2010). Developing Country Business Cycles: Revisiting the Stylised facts, Working Paper, School of Economics and Finace, Universidad de Londres, Londres.

Mérida, A. y Golpe, A. G. (2016). Tourism-leg Growth Revisited for Spain: Causality, Business Cycles and Structural Breaks, International Journal of Tourism Research, 18: 39-51.

Nowak, J.J.; Sahli, M. y Cortés-Jiménez, I. (2007). Tourism, capital goods, imports and economic grown: theory and evidence for Spain, Tourism Economic, 13 (4), 515-536,

OECD (2009). The World competitiveness Report. Lausan, Foro Económico Mundial.

OMT (2001). Global Forecasts and profiles of market segments. Tourism 2020 Vision, Vol. 7, Organización Mundial del Turismo Madrid.

Pedreño Muñoz, A. (dir.) (1996). Introducción a la economía del turismo en España, Civitas, Madrid.

Perles-Ribes, J.F., Ramón-Rodríguez, Rubia, A. y Moreno-Izquierdo, L. (2017). Is the tourism-led growth hypothesis valid after the global economic and financial crisis? The case of Spain 1957-2014), Tourism Management, 61, 96-109.

Pierce, D. A. y Haugh, L. (1977). Causality in Temporal System: Characterization and Survey, Journal of Econometrics, 5(3), 265-293.

Peña, D. (2010). Análisis de Series Temporales, Alianza.

Pierce, D.A. y Haugh, L. D. (1977). Causality in temporal systems: Characterization and a survey, Journal of Econometric, 265-293.

Pulido, J.I., Flores, D. y Vargas, M.J. (2008): Gestión activa de la deuda externa y desarrollo turístico. Los swaps deuda-turismo sostenible, Revista de Economía Mundial, 20, 197-227

Sinclair, M.T. (1998). Tourism and Economic Development: A Survey, The Journal of Development Studies, 34(5), 1-51.

Torres Bernier, E. (1994). Turismo y desarrollo regional, Papers de Turisme, 14-15, 95-102.

Tribe, J. (2005): The Economics of Recreation, Leisure and Tourism, Oxford, Elservier.

WTTC (2005): Progress and Priorities 2005-06, Reino Unido, World Travel and Tourism Council. 
PIB

Número de Viajes. Turismo Emisor.

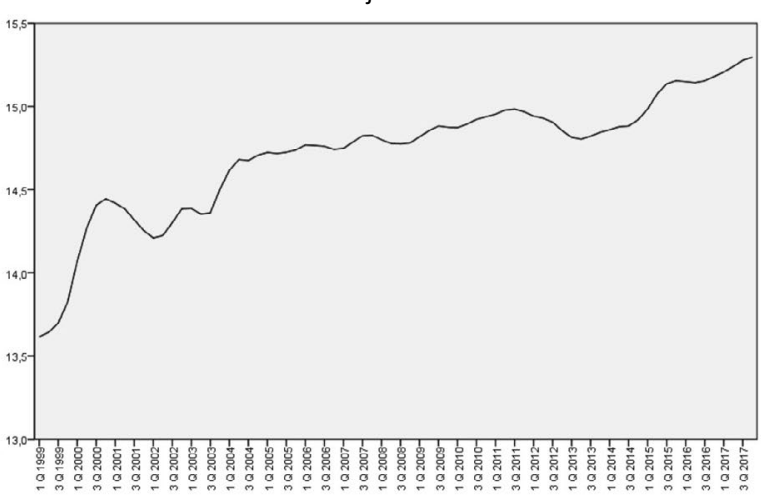

Número de Viajes. Turismo Interno.

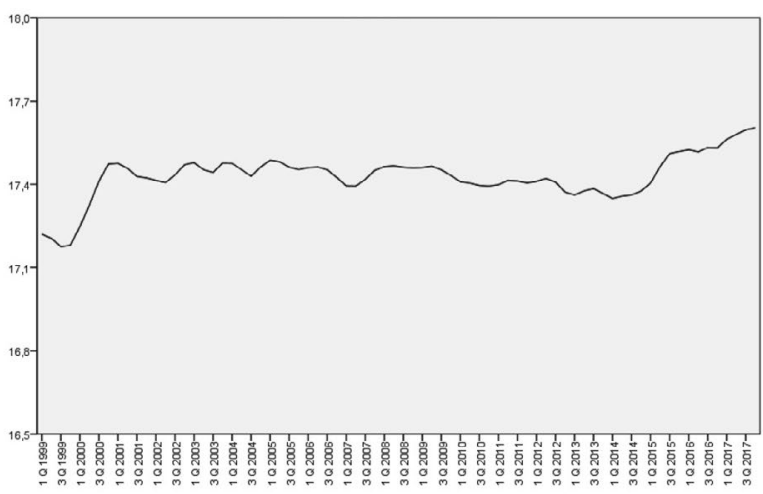

Número de Viajes. Turismo Nacional.

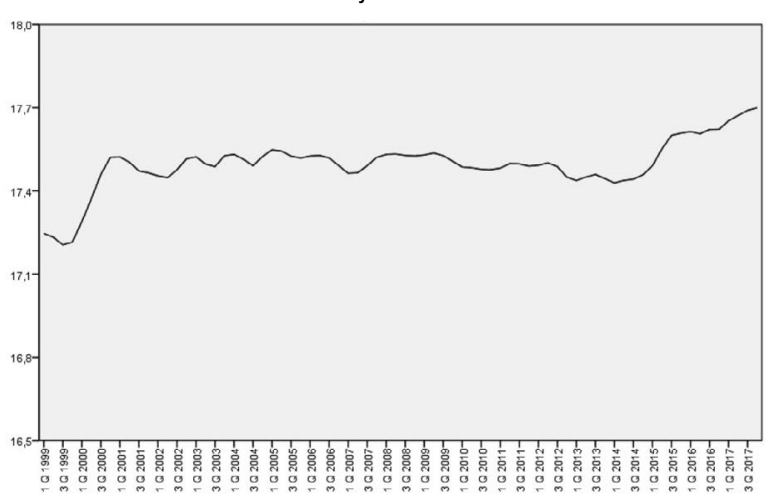

PIB

Número de Viajes. Turismo Emisor.

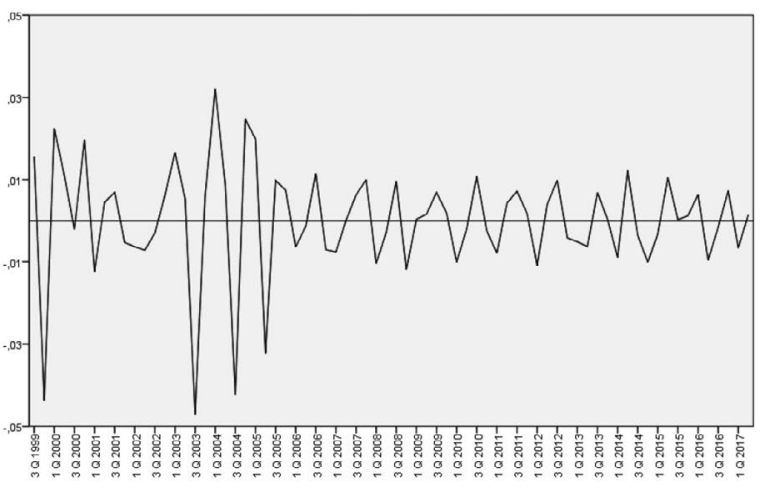

Número de Viajes. Turismo Interno.

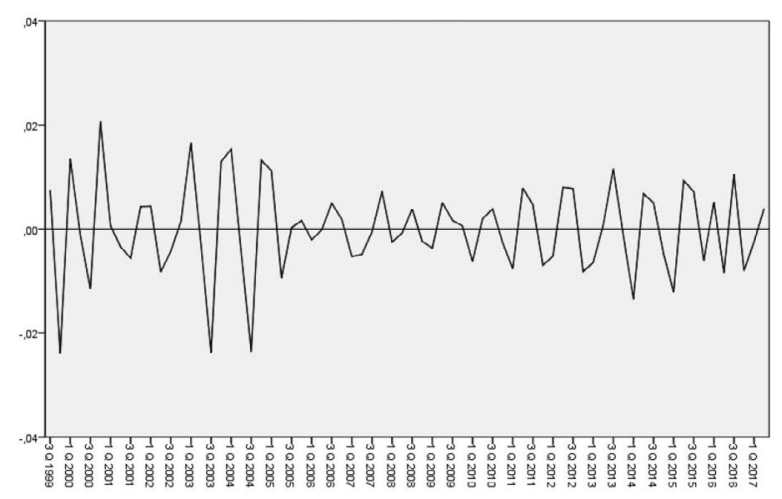

Número de Viajes. Turismo Nacional.

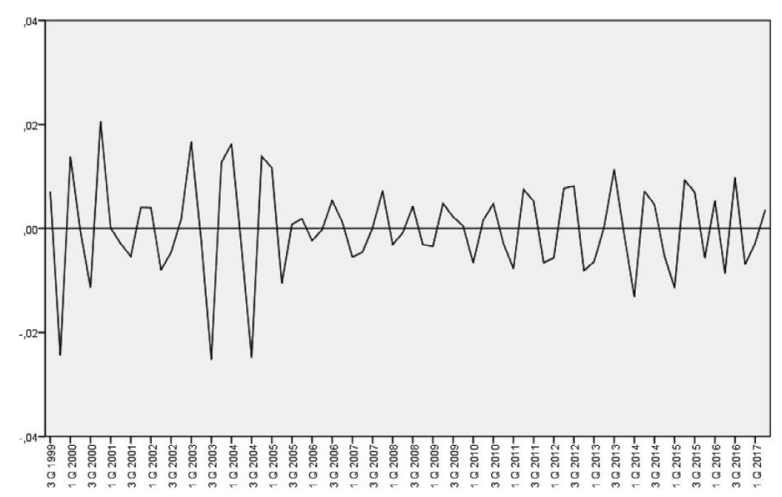

Figura 1. Gráficos de las series temporales desestacionalizadas y de la componente cíclica. 
PIB

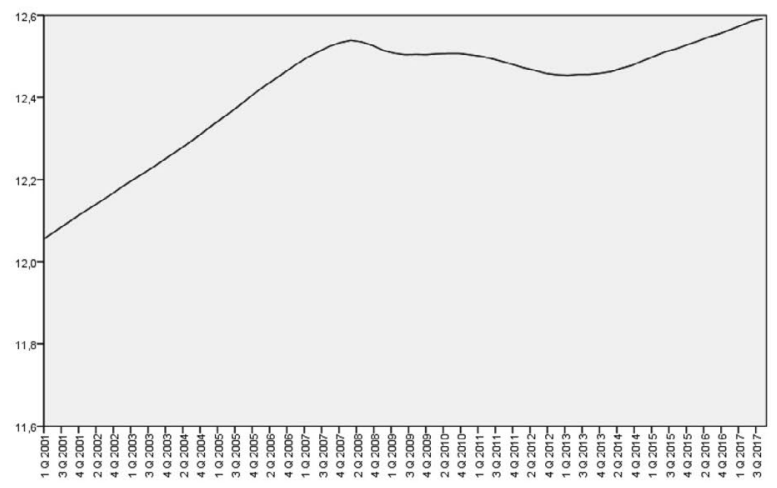

Pernoctaciones en hoteles.

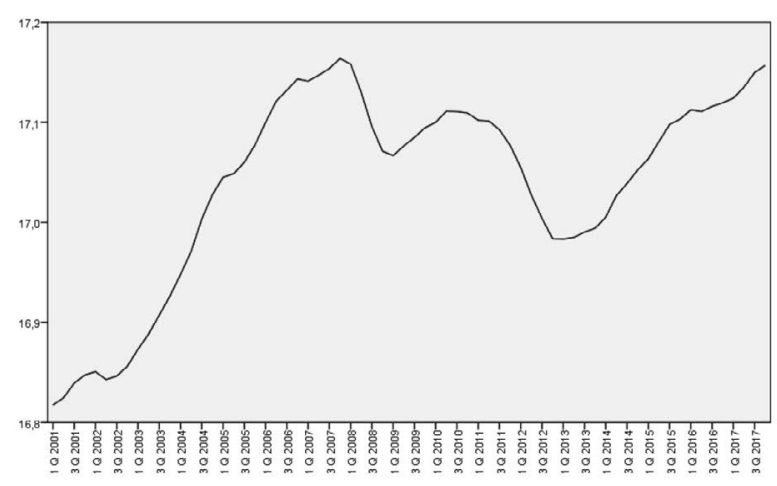

Pernoctaciones en alojamientos rurales.

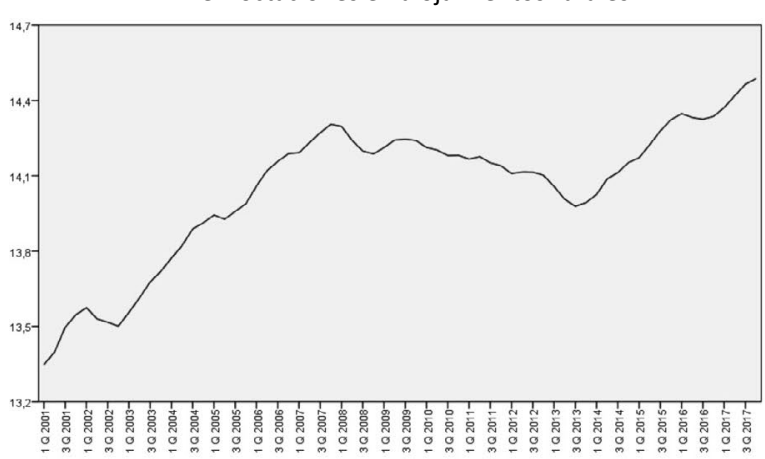

Suma pernoctaciones en hoteles y alojamientos rurales.

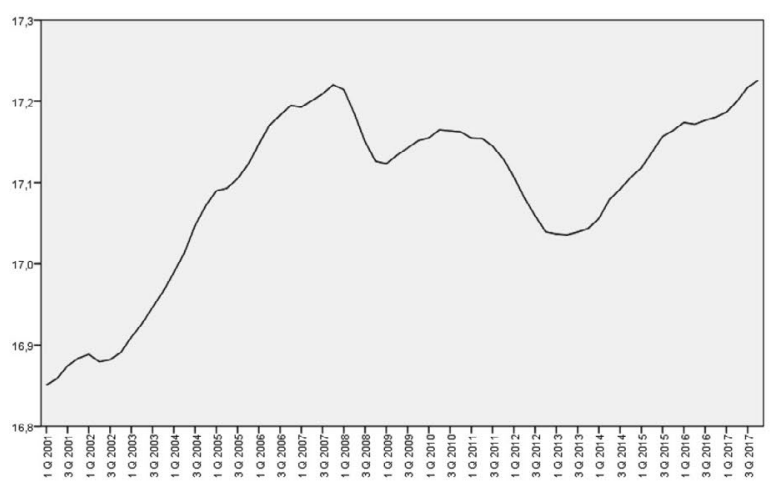

PIB

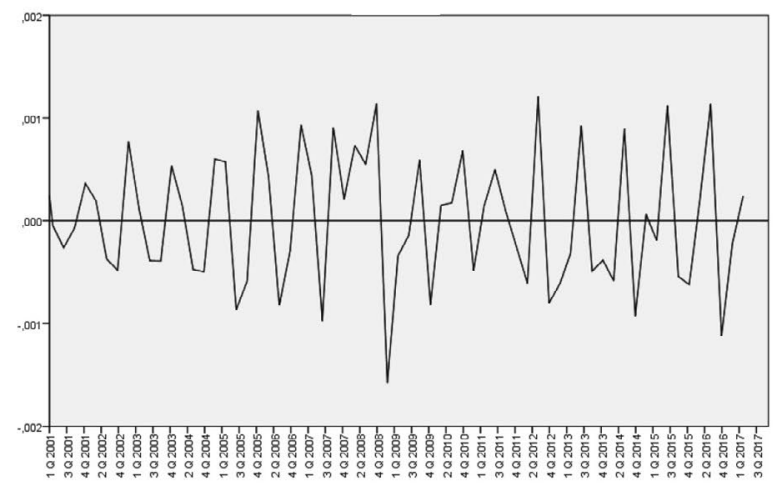

Pernoctaciones en hoteles.

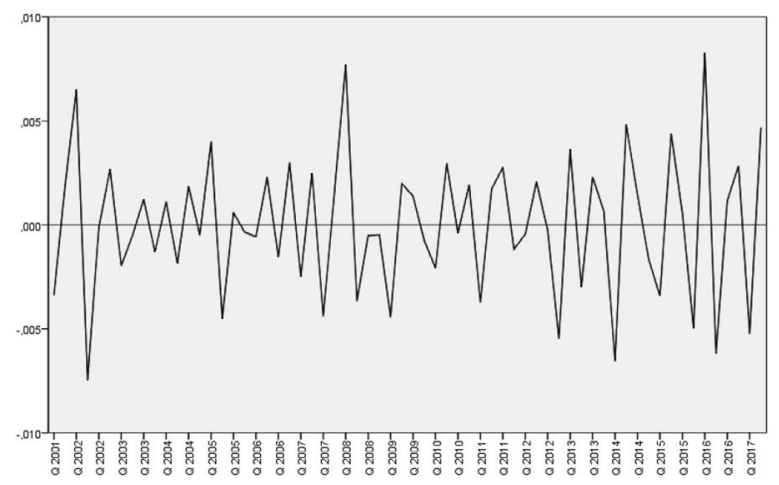

Pernoctaciones en alojamientos rurales.

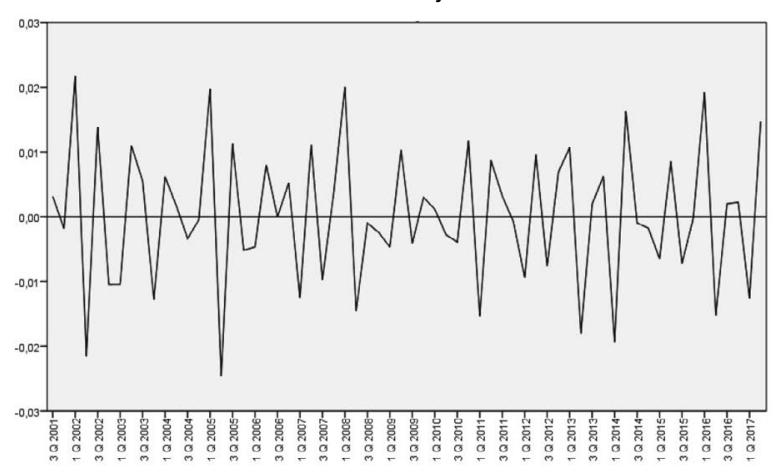

Suma pernoctaciones en hoteles y alojamientos rurales.

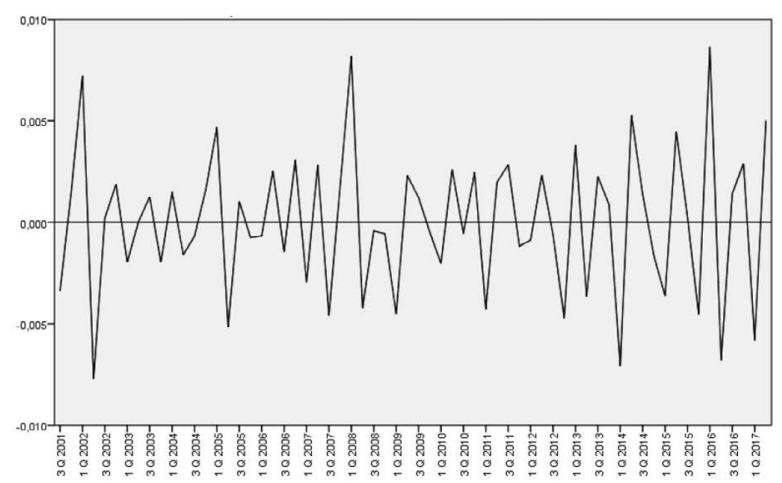

Figura 1. Gráficos de las series temporales desestacionalizadas y de la componente cíclica. 